\title{
SENSITIVE DEPENDENCE ON PARAMETERS
}

\author{
MICHAE MISIUREWICZ
}

\begin{abstract}
For a family of dynamical systems we define sensitive dependence on parameters in a way resembling Guckenheimer's definition of sensitive dependence on initial conditions. While sensitive dependence on initial conditions tells us that if we know the initial condition only approximately then we cannot make deterministic predictions, sensitive dependence on parameters tells us that if we know the parameter value only approximately then we cannot make statistical predictions. We show that the family of logistic maps has sensitive dependence on parameters. Moreover, the long-time response of the system to the change of the parameter is practically independent of the immediate response.
\end{abstract}

\section{INTRODUCTION}

It has been well known for a long time that deterministic systems can be chaotic, with no chances for making accurate long-term predictions. This is due to the fact that many systems have sensitive dependence on initial conditions. This notion has been introduced in 1979 by John Guckenheimer [3] (without a precise definition, it has been used slightly earlier by David Ruelle [7]). We will define it in the next section. For now, let us just say that it means that there is a large subset of the phase space, such that a trajectory starting at a point of this subset can be significantly changed by an arbitrarily small change of the starting point. Since we cannot measure the starting point with an infinite precision, this means that we cannot predict the long-term behavior of the trajectory.

This means that the only chance of saving the idea of predictability is to make statistical predictions. If we measure the values of some observable (a continuous function on the phase space), it may be impossible to predict exactly its long-term fluctuations, but it may be possible to predict the limit of its time averages, if not everywhere, then at least almost everywhere. This may be due to the existence of the Sinai-Ruelle-Bowen (SRB) ergodic invariant measure. Then the time averages of the observable converge almost everywhere to its space average, as the time goes to infinity.

We will show that it may happen that this idea does not work either, due to the sensitive dependence on parameters. Indeed, not only we cannot measure exactly the initial conditions of the system; we also cannot measure exactly the values of the parameters on which the mapping that we iterate depends. It can happen that arbitrarily small changes of the parameters can result in significant changes of the SRB measure, and as a consequence, in significant changes of the averages of our observable.

Date: April 17, 2010.

2000 Mathematics Subject Classification. 37E05, 37C40.

Key words and phrases. Sensitive dependence, chaos, logistic maps. 
As an illustration of the above phenomenon, we will consider the popular family of logistic maps $f_{r}(x)=r x(1-x)$ of the unit interval to itself and the simplest nontrivial observable, the identity. This is the same family that motivated Guckenheimer to define sensitive dependence on initial conditions.

In fact, our example illustrates also another problem with predictability. As the parameter $r$ increases, the immediate effect on the observable is that it increases: $s>r$ implies $f_{s}(x)>f_{r}(x)$ for all $x \in(0,1)$. However, the long-time effect is kind of random; sometimes the averages increase and sometimes decrease.

The paper is organized as follows. In Section 2, basic definitions are provided. In Section 3, necessary properties of the family of logistic maps are recalled. In Section 4, the technical part of the work, using kneading theory, is done. In Section 5, the work is continued on the level of invariant measures and integrals of observables. The section completes the proof of the main result of the paper, that the family of logistic maps has sensitivity on parameters. Section 7 contains some generalizations. Moreover, several points of view on sensitivity are presented.

\section{Preliminaries}

We will consider a metric compact space $X$ (with metric $d$ ) and a continuous map $f: X \rightarrow X$. We will sometimes refer to the pair $(X, f)$ as a dynamical system or just system; the space $X$ will be called the phase space. For $n \geq 0$ the map $f^{n}$ is the $n$-th iterate of $f$. We also consider a certain natural probability measure on $X$, which we will call the reference measure. If we speak about a measure on $X$ without specifying which measure it is, we mean the reference measure. As we mentioned, this measure is "natural." In particular, if $X$ is a subset of $\mathbb{R}^{n}$, then this is a (normed) Lebesgue measure. If $X$ is a Riemannian manifold, then this is the (normed) Riemann measure, etc.

Definition 2.1 (Guckenheimer, [3]). The system $(X, f)$ has sensitive dependence on initial conditions if there is a set $Y \subset X$ of positive measure and $\varepsilon>0$ such that for any $x \in Y$ and neighborhood $U$ of $x$ there is $y \in U$ and $n \geq 0$ with $d\left(f^{n}(x), f^{n}(y)\right)>\varepsilon$.

We will mimic this definition for parameters instead of initial conditions. Moreover, as we mentioned in the introduction, we will look at the averages of an observable rather than at individual trajectories. Therefore we have to prepare the ground for the new definition.

There are several distinct definitions of the SRB measure. Since we need its uniqueness, we choose the following one. By $\delta_{x}$ we denote the Dirac delta measure at $x$, that is, the probability measure concentrated at $x$.

Definition 2.2. A probability measure $\sigma$ on $X$ is called an $S R B$ measure for $f$ if for almost every (with respect to the reference measure) $x \in X$ the averages

$$
\frac{1}{n} \sum_{k=0}^{n-1} \delta_{f^{k}(x)}
$$

converge to $\sigma$ in the weak- $*$ topology as $n \rightarrow \infty$. 
With this definition, the SRB measure, if it exists, is automatically invariant, ergodic and unique. Moreover, by the definition of the weak-* topology, the averages

$$
\frac{1}{n} \sum_{k=0}^{n-1} \varphi\left(f^{k}(x)\right)
$$

converge to $\int \varphi d \sigma$ as $n \rightarrow \infty$ for every observable $\varphi: X \rightarrow \mathbb{R}$ and almost every (with respect to the reference measure) $x \in X$.

Now we deal with the following situation. We consider a family of maps $f_{t}: X \rightarrow X$, parametrized by $t \in T$. In $T$ we have topology and some reference parameter measure. Usually $T$ is some nice subset of an Euclidean space, most often an interval in $\mathbb{R}$, and then this measure is the Lebesgue measure. We assume that for a subset $T^{\prime} \subset T$ of positive measure there exists an SRB measure $\sigma_{t}$ for $\left(X, f_{t}\right)$.

Definition 2.3. The family $\left(X, f_{t}\right)$ has sensitive dependence on parameters if there exists an observable $\varphi: X \rightarrow \mathbb{R}$, a set $S \subset T^{\prime}$ of positive measure and $\varepsilon>0$ such that for any $s \in S$ and a neighborhood $U$ of $s$ there is $t \in U \cap T^{\prime}$ with

$$
\left|\int \varphi d \sigma_{s}-\int \varphi d \sigma_{t}\right|>\varepsilon .
$$

\section{LOGISTIC MAPS}

Let us recall some facts about the family of logistic maps. As we mentioned, they are maps $f_{r}:[0,1] \rightarrow[0,1]$ given by the formula $f_{r}(x)=r x(1-x)$, where $0<r \leq 4$. For $r \leq 3$ the dynamics of $f_{r}$ is not interesting; all trajectories (except perhaps those of ) and 1) converge to an attracting or neutral fixed point. Therefore we will consider only $r \in(3,4]$. Then the interval $M_{r}=\left[f_{r}^{2}(c), f_{r}(c)\right]$, where $c=1 / 2$ is the unique critical point of $f_{r}$, is invariant. Moreover, the only nonwandering point of $f_{r}$ outside this interval is the fixed point 0 .

The family of logistic maps (or equivalent to it via simple affine conjugacies, family of quadratic maps $x \mapsto x^{2}+a$ ) is the most popular one-parameter family of interval maps, so a lot is known about it. We will list the properties that we will need later. In the next section, we will add two more properties, connected with the kneading theory.

To state some of the properties we need more terminology and notations. A map $f_{r}$ is renormalizable if there exist a finite set of pairwise disjoint open intervals $J_{1}, \ldots, J_{n} \subset[0,1]$ such that $f\left(J_{1}\right) \subset J_{2}, \ldots, f\left(J_{n-1}\right) \subset J_{n}, f\left(J_{n}\right) \subset J_{1}$, and $n \geq 2$. Otherwise, $f_{r}$ is non-renormalizable. Let us denote by $\mathcal{N}$ the set of those $r \in(3,4]$ for which $f_{r}$ is non-renormalizable.

Note that if $f_{r}$ has an attracting or neutral periodic orbit of period larger than 1 , then, according to our definition, $f_{r}$ is renormalizable. Since we are assuming that $r \in(3,4]$, the map $f_{r}$ has no attracting or neutral fixed point.

A tent map with slope $s \in(0,2]$ is a piecewise linear map of the interval $[0,1]$ to itself, given by $x \mapsto s(1 / 2-|x-1 / 2|)$.

Theorem 3.1. The family $f_{r}(r \in(3,4])$ of logistic maps has the following properties.

(a) If the critical point of $f_{r}$ is eventually periodic but not periodic, then the preimages (under the iterates of $f_{r}$ ) of $c$ are dense in $M_{r}$, and all periodic orbits of $f_{r}$ are repelling. 
(b) If $r \in \mathcal{N}$ then $f_{r}$ is transitive.

(c) If $r \in \mathcal{N}$ then $\left.f_{r}\right|_{M_{r}}$ is conjugate to exactly one tent map $g$ with slope $s(r) \in$ $[\sqrt{2}, 2]$ restricted to the interval $\left[g^{2}(1 / 2), g(1 / 2)\right]$, with the trajectory of $1 / 2$ not periodic. This map $r \mapsto s(r)$ is onto and continuous.

(d) The set $\mathcal{N}$ has positive Lebesgue measure.

(e) For almost every $r \in(3,4]$ the map $f_{r}$ has an $S R B$ measure.

Proof. Properties (a), (b) and (c) are well known (see, e.g., [6], [2]). Property (e) can be found in [5]. Property (d), although kind of well known, is surprisingly difficult to find in the literature. Probably the best approach is to observe that the standard proofs of the existence of absolutely continuous $f_{r}$-invariant measure for a set of $r$ 's of positive measure give an ergodic measure with the support equal to $M_{r}$ (see, e.g., [8]). Thus, those values of $r$ belong to $\mathcal{N}$.

Of course, the reference measure for logistic maps, both in the phase space and in the parameter space, is the Lebesgue measure.

We will denote by $\mathcal{S}$ the set of those parameters $r \in(3,4]$ for which the SRB measure for $f_{r}$ exists, and we will denote this measure by $\sigma_{r}$.

\section{KNEADING}

There are several approaches to the kneading theory. We will use the one presented in [2].

In the kneading theory one considers kneading sequences and itineraries for unimodal maps. They are sequences, finite or infinite, of symbols $L, C, R$. If the sequence is finite, then it ends in $C$. Moreover, the symbol $C$ can appear only at the end of a finite sequence. The space of all such sequences will be denoted by $\Sigma$. There is the shift map $\sigma: \Sigma \rightarrow \Sigma$, defined in a usual way. Namely, we shift a sequence by one term to the left, and this leads to deleting the first term of the sequence. For instance, $\sigma(R L R L L R L L L \ldots)=L R L L R L L L \ldots$. Finite sequences become shorter under the action of $\sigma$; under the iterates of $\sigma$ they eventually become the empty sequence.

We will refer to finite sequences of symbols $L, R$ as blocks. Writing blocks one after another means concatenation, so for instance, if $A=R L$ and $B=R R R L L$, then $A B=R L R R R L L, A^{3}=R L R L R L$, and $A^{\infty}=R L R L R L R L \ldots$. For a block $A$ we will denote by $|A|$ its length. We will also sometimes apply $\sigma$ to blocks.

For a unimodal map $f:[0,1] \rightarrow[0,1]$, increasing on $[0, c]$ and decreasing on $[c, 1]$, the itinerary of a point $x$ is a sequence $A_{0} A_{1} A_{2} \ldots$, where $A_{i}=L$ if $f^{i}(x)<c$ and $A_{i}=R$ if $f^{i}(x)>c$ (and $A_{i}=C$ if $f^{i}(x)=c$; then the itinerary terminates). The itinerary of $f(c)$ is called the kneading sequence of $f$. The order of the points on the interval is the same as the order of their itineraries, which is defined as follows. First we order symbols $L<C<R$. Now, given two distinct sequences $A=A_{0} A_{1} A_{2} \ldots$, $B=B_{0} B_{1} B_{2} \cdots \in \Sigma$, we find the smallest index $n$ for which $A_{n} \neq B_{n}$. If the number of $R$ 's among $A_{0}, \ldots, A_{n-1}$ is even, then $A_{n}<B_{n}$ means $A<B$, and $A_{n}>B_{n}$ means $A>B$. If the number of $R$ 's among $A_{0}, \ldots, A_{n-1}$ is odd, the inequalities are reversed. We will also use a similar order on the blocks of equal length. If the itinerary of $x$ is $A \neq C$ then the itinerary of $f(x)$ is $\sigma(A)$.

For all points $x \in\left[f^{2}(c), f(c)\right)$ we have $x<f(c)$, so the itinerary of $x$ is smaller than the kneading sequence of $f$. In particular, if the kneading sequence of $f$ is $K$, 
then $\sigma^{n}(K)<K$ for all $n \geq 1$ (we consider the empty sequence to be smaller than all other sequences).

There are two types of completeness. The first one applies to a given unimodal map $f$. If $K$ is a kneading sequence of $f$ and a sequence $A \in \Sigma$ is such that $\sigma^{n}(A)<K$ $\left(\sigma^{n}(A)<\min \left((D L)^{\infty},(D R)^{\infty}\right)\right.$ if $\left.K=D C\right)$ for all $n \geq 0$, then there is a point $x \in\left[f^{2}(c), f(c)\right]$ with the itinerary $A$. The second kind of completeness applies to some families of maps, in particular to the family of the logistic maps. If a sequence $K \in \Sigma$ is admissible (in [2] the term "maximal" is used; "admissible" has different meaning there), that is, it starts with $R$ and $\sigma^{n}(K) \leq K$ for all $n$ (for all $n \leq|D|$ if $K=D C$ ), then there is a map $f$ from that family with the kneading sequence $K$.

Let us start with two more properties of logistic maps, promised in the preceding section.

Lemma 4.1. The family $f_{r}(r \in(3,4])$ of logistic maps has the following properties.

(a) If the kneading sequence of $f_{r}$ is eventually periodic but not periodic, then the orbit of $c$ is eventually periodic but not periodic.

(b) If $r \in \mathcal{N}$ then for every $\varepsilon>0$ there is $n$ such that if the kneading sequences of $f_{r}$ and $f_{s}$ coincide on the initial block of length $n$ then $|s-r|<\varepsilon$.

Proof. (a) By a theorem from [2, page 69], if the kneading sequence of $f_{r}$ is eventually periodic but not periodic, then all periodic orbits of $f_{r}$ are repelling (so, in particular the trajectory of $c$ cannot be periodic). However, then the periodic part of the kneading sequence of $f_{r}$ is an itinerary of a unique point, so this point has to be periodic. Therefore some image of $c$ is periodic.

(b) The dependence of the kneading sequence on the parameter is monotone and if $r \in \mathcal{N}$, then no other $f_{s}$ has the same kneading sequence as $f_{r}$ (see, e.g., [6]). However, negation of the statement (b) of the lemma would imply existence of $s$ such that $f_{s}$ has the same kneading sequence as $f_{r}$ and $|s-r| \geq \varepsilon$, a contradiction.

The next lemma is stated in the terms of kneading sequences and itineraries. From the point of view of the family of logistic maps it is a result of the "closing lemma" type. The interpretation is that if a repelling periodic point is in the $\omega$-limit set of the critical point then by a slight change of the parameter we can make the trajectory of the critical point to fall into this point.

Lemma 4.2. Let $A$ be a block and let $K \in \Sigma$ be an infinite admissible sequence in which blocks $A^{k}$ appear for every $k$ and which is not periodic. Then for every $m$ there is an initial block $D$ of $K$, such that $|D| \geq m$ and the sequence $D A^{\infty}$ is admissible and not periodic.

Proof. If $K$ is of the form $B A^{\infty}$ for some block $B$, then we can take $D=B A^{m}$, and then $|D| \geq m$ and $K=D A^{\infty}$.

Assume now that $K$ is not of the form $B A^{\infty}$. For every $k$ there exists $D_{k}$ such that $K$ begins with $D_{k} A^{k}$ and this occurrence of $A^{k}$ is the first one in $K$. Since $K$ is not of the form $B A^{\infty}$, the length of $D_{k}$ goes to infinity as $k \rightarrow \infty$. Therefore, there is $k$ such that

(a) $\left|D_{k}\right| \geq m$

(b) $K$ does not begin with $\sigma^{i}\left(A^{k}\right)$ for $i=0,1, \ldots,|A|-1$. 
Set $D=D_{k}$. Then $K$ begins with $D A^{k}$ and there is only one place in $D A^{k}$ where $A^{k}$ occurs.

Compare $\sigma^{n}\left(D A^{\infty}\right)$ with $D A^{\infty}$. Assume first that $n \leq|D|$ (see Figure 1$)$. Then the block $\sigma^{n}\left(D A^{k}\right)$ must be different than the initial block $F$ of $D A^{\infty}$ of the same length, because otherwise we would have an additional occurrence of the block $A^{k}$ in $D A^{k}$. However, the blocks $\sigma^{n}\left(D A^{k}\right)$ and $F$ are initial blocks of $\sigma^{n}(K)$ and $K$ respectively. Thus, since $\sigma^{n}(K)<K$, we get $\sigma^{n}\left(D A^{k}\right)<F$. Therefore $\sigma^{n}\left(D A^{\infty}\right)<D A^{\infty}$.

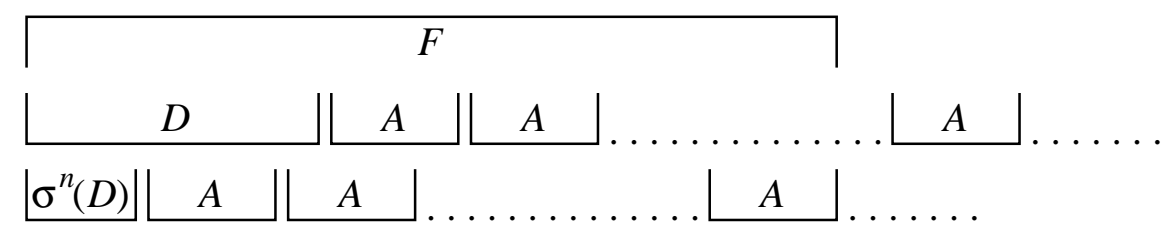

Figure 1. Case $n \leq|D|$ from the proof of Lemma 4.2 .

Assume now that $n>|D|$ (see Figure 2). Then $\sigma^{n}\left(D A^{\infty}\right)$ begins with $\sigma^{i}\left(A^{k}\right)$ for some $i \in\{0,1, \ldots,|A|-1\}$. By (b), this block is different than the initial block $F$ of $D A^{\infty}$ of the same length, and therefore, $\sigma^{n}\left(D A^{\infty}\right)<F$. The blocks $\sigma^{i}\left(A^{k}\right)$ and $F$ are initial blocks of $\sigma^{|D|+i}(K)$ and $K$ respectively, and we get $\sigma^{n}\left(D A^{\infty}\right)<D A^{\infty}$ as in the preceding paragraph.

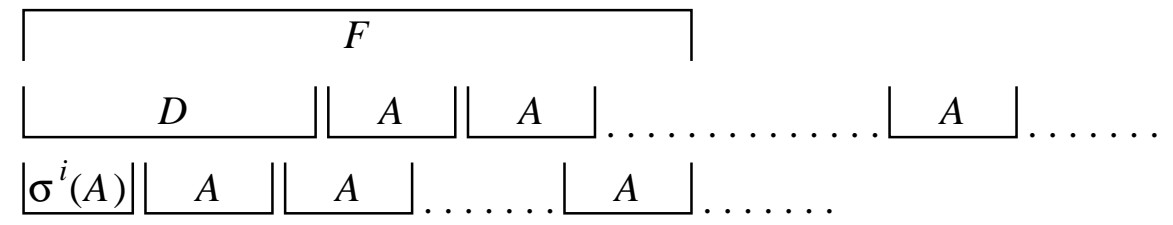

Figure 2. Case $n>|D|$ from the proof of Lemma 4.2 .

The sequence $D A^{\infty}$ is not periodic because, as we already noticed, for every $i \in$ $\{0,1, \ldots,|A|-1\}$ the block $\sigma^{i}\left(A^{k}\right)$ is not an initial block of $D A^{\infty}$.

The next lemma is again a "closing lemma" type result. If the trajectory of the critical point falls into a periodic orbit, then with a small perturbation of the parameter we can close the orbit of the critical point, in such a way that it stays very close to this periodic orbit for most of the time.

Lemma 4.3. Let $A$ and $D$ be blocks such that the sequence $D A^{\infty}$ is admissible and not periodic. Then there exists a block $E$ and a number $m$ such that for every $k \geq m$ the sequence $D A^{k} E C$ is admissible.

Proof. There exists a map $f_{r}$ from the logistic family with the kneading sequence $D A^{\infty}$. This sequence is eventually periodic but not periodic, so by Lemma 4.1 (a), the critical point $c$ of $f_{r}$ is eventually periodic but not periodic. Therefore, by Theorem 3.1 (a), preimages of $c$ are dense in $M_{r}$. We have $\sigma^{n}\left(A^{\infty}\right)<D A^{\infty}$ for all $n$, so 
there exists a point $a \in\left[0, f_{r}(c)\right]$ with itinerary $A^{\infty}$. The itinerary is periodic and by Theorem 3.1 (a), $f_{r}$ has no attracting or neutral periodic points, so $a$ is a repelling periodic point.

Since $a$ is periodic, then either $a \in M_{r}$ or $a=0$. If $a=0$ then $A=L^{k}$ for some $k$, so $D A^{\infty}=D L^{\infty}$. This means that the trajectory of $c$ falls eventually into 0 . This is possible only if $r=4$. Then $f_{r}^{2}(c)=0$, so in this case also $a \in M_{r}$.

Since $a$ is repelling, if we choose a point $x_{0} \in M_{r}$ sufficiently close to $a$, we can find points $x_{1}, x_{2}, \ldots$ closer and closer to the corresponding preimages of $a$ along its periodic orbit, and then $x_{n}$ will lie on the same side of $c$ as the corresponding preimage of $a$. For each $j \geq 0$ the itinerary of $x_{j n}$ is $A^{j} E C$, where $E C$ is the itinerary of $x_{0}$. This proves that $\sigma^{i}\left(A^{j}\right) E C<D A^{\infty}$ for all $j>0$ and $i \in\{0,1, \ldots,|A|-1\}$.

Take $m=\max \{|D|,|E|+1\} /|A|+1$. If $k \geq m$ then $\left|A^{k-1}\right|=(k-1)|A| \geq$ $|E|+1=|E C|$. We will show that then the sequence $D A^{k} E C$ is admissible. For $n \geq 1$ compare $\sigma^{n}\left(D A^{k} E C\right)$ with $D A^{k} E C$. Assume first that $n \leq\left|A^{k-1}\right|$ (see Figure 3 ). Then the initial block $F$ of $\sigma^{n}\left(D A^{k} E C\right)$ of length $|D A|$ is the same as the initial block of $\sigma^{n}\left(D A^{\infty}\right)$ of this length. Therefore $F \leq D A$. We can write $F=G H$ where $|G|=|D|$ and $|H|=|A|$. Then $H=\sigma^{i}(A)$ for some $i \in\{0,1, \ldots,|A|-1\}$ and we get $\sigma^{n}\left(D A^{\infty}\right)=G H^{\infty}$. If $F=D A$, then $G=D$ and $H=A$, so $\sigma^{n}\left(D A^{\infty}\right)=D A^{\infty}$, a contradiction. Therefore $F<D A$. This proves that $\sigma^{n}\left(D A^{k} E C\right)<D A^{k} E C$.

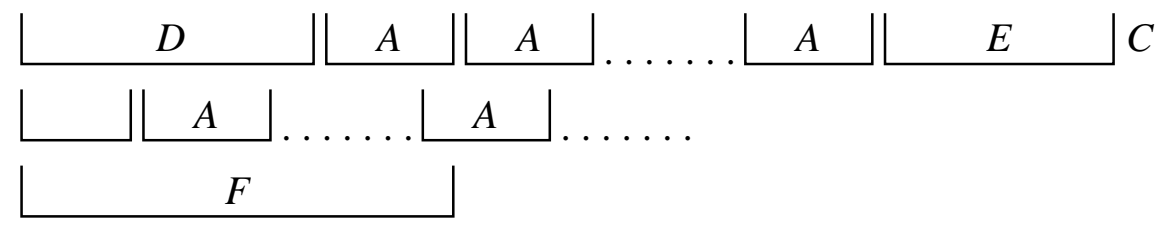

Figure 3. Case $n \leq\left|A^{k-1}\right|$ from the proof of Lemma 4.3.

Assume now that $\left|A^{k-1}\right|<n<\left|D A^{k}\right|$. Since $k \geq m$, we have $n>\left|A^{k-1}\right|=$ $(k-1)|A| \geq|D|$. Hence, $\sigma^{n}\left(D A^{k} E C\right)$ is of the form $\sigma^{i}\left(A^{j}\right) E C$ for some $j>0$ and $i \in\{0,1, \ldots,|A|-1\}$. Therefore, as we proved, $\sigma^{n}\left(D A^{k} E C\right)<D A^{\infty}$. Moreover, $n>\left|A^{k-1}\right|=(k-1)|A| \geq|E C|$, so $\left|\sigma^{n}\left(D A^{k} E C\right)\right|<\left|D A^{k}\right|$, and thus $\sigma^{n}\left(D A^{k} E C\right)<$ $D A^{k} E C$.

Assume finally that $n \geq\left|D A^{k}\right|$. Then $\sigma^{n}\left(D A^{k} E C\right)=\sigma^{i}(E C)$ for some $i \geq 0$. Since $E C$ is the itinerary of $x_{0}$ for the map $f_{r}$, whose kneading sequence is $D A^{\infty}$, we have $\sigma^{i}(E C)<D A^{\infty}$. As in the preceding paragraph, we get $\sigma^{n}\left(D A^{k} E C\right)<D A^{k} E C$. This completes the proof.

If $Q$ is a finite subset of $[0,1]$ then by $\mu_{Q}$ we will denote the probability measure equidistributed on $Q$. When measuring distance between periodic orbits $P$ of $f$ and $Q$ of $g$ of the same period $m$, we choose points $x \in P$ and $y \in Q$, then take the maximum of $\left|f^{i}(x)-g^{i}(y)\right|$ over $i=0,1, \ldots, m-1$, and then take the minimum over the choices of $y \in Q$.

Theorem 4.4. Assume that $r \in \mathcal{N}$ and let $P$ be a periodic orbit of $f_{r}$ contained in $M_{r}$. Then there is a sequence $\left(r_{n}\right)$ convergent to $r$ such that for each $n$ the $f_{r_{n}}$-orbit $P_{n}$ of $c$ is periodic and the measures $\mu_{P_{n}}$ converge to $\mu_{P}$ in the weak-* topology. 
Proof. By Theorem 3.1 (c), the map $f_{r}$ is conjugate to a tent map $g$ with the slope in $(\sqrt{2}, 2]$. By [1], arbitrarily close to $g$ there is a tent map $\widetilde{g}$ for which the trajectory of the turning point $c=1 / 2$ is dense in $\left[\widetilde{g}^{2}(c), \widetilde{g}(c)\right]$. Again by Theorem 3.1 (c), the map $\widetilde{g}$ is conjugate to a logistic map $f_{s}$, and closeness of $g$ and $\widetilde{g}$ translates into closeness of $r$ and $s$. That is, as the slope of $\widetilde{g}$ approaches the slope of $g$, then $s$ approaches $r$. This proves that arbitrarily close to $r$ there are parameters $s$ such that the $f_{s}$-trajectory of $c$ is dense in $M_{r}$.

Points of the orbit $P$ of $f_{r}$ are zeros of a certain polynomial in $x$, whose coefficients are continuous functions of the parameter $r$. Thus, for the parameters $t$ sufficiently close to $r$ there are periodic orbits $P(t)$, depending continuously on $t$, and such that $P(r)=r$ (note that the assumptions on $f_{r}$ rule out a bifurcation of $P(t)$ at $t=r$ ).

Consider the map $f_{s}$. Choose one point $x \in P$. The itinerary of $x$ is of the form $A^{\infty}$ for some block $A$. The trajectory of $c$ comes arbitrarily close to the orbit $P$, so for every $k$ the block $A^{k}$ appears in the kneading sequence of $f_{s}$. Moreover, since the trajectory of $c$ is dense, the kneading sequence $K$ of $f_{s}$ cannot be finite or periodic. Thus, by Lemma 4.2 , for every $m$ there is an initial block $D$ of $K$, such that $|D| \geq m$ and the sequence $D A^{\infty}$ is admissible and not periodic. For each such $D$ there is a parameter $s_{D}$ such that the map $f_{s_{D}}$ has the kneading sequence $D A^{\infty}$. Moreover, as $|D|$ goes to infinity, the sequences $D A^{\infty}$ and $K$ coincide on longer and longer initial blocks, so by Lemma 4.1 (b), $s_{D}$ converges to $s$. Thus, we may assume that $s_{D}$ is as close to $s$ as we want.

In the same way as above, but using Lemma 4.3 rather than Lemma 4.2 , we find for every sufficiently large $k$ a parameter $s_{k}$ such that the kneading invariant of $f_{s_{k}}$ is $D A^{k} E C$. Moreover, by taking $k$ as large as necessary, we may make $s_{k}$ as close to $s_{D}$ as we want.

Let us summarize what we achieved up to now. We proved that for every $\varepsilon>0$ there exist blocks $D, E$ such that for every sufficiently large $k$ there is $s_{k}$ with $\left|s_{k}-r\right|<\varepsilon$ and such that the kneading sequence of $f_{s_{k}}$ is $D A^{k} E C$. Moreover, the distance between the periodic orbits $P$ of $f_{r}$ and $P\left(s_{k}\right)$ of $f_{s_{k}}$ is smaller than $\varepsilon$. The $f_{r}$-itinerary of the point $x \in P$ is $A^{\infty}$, and thus, if $\varepsilon$ is sufficiently small, then the $f_{s_{k}}$-itinerary of the corresponding point $x\left(s_{k}\right) \in P\left(s_{k}\right)$ is also $A^{\infty}$. As $k \rightarrow \infty$, the parameters $s_{k}$ converge to a parameter $s_{D}$, for which the orbit $P\left(s_{D}\right)$ is repelling.

Since the $f_{s_{D}}$-orbit $P\left(s_{D}\right)$ is repelling, there is $m$ such that if a point $z$ has the $f_{s_{D}}$-itinerary beginning with $A^{k}$ and $k>m$ then $\left|f_{s_{D}}^{i}(z)-f_{s_{D}}^{i}(x)\right|<\varepsilon$ for $0 \leq i<$ $(k-m)|A|$. Since $s_{k} \rightarrow s_{D}$, the same is true for $s_{k}$ replacing $s_{D}$ if $k$ is sufficiently large. Then a segment of the periodic $f_{s_{k}}$-orbit $Q_{k}$ of $c$ of length at least $(k-m)|A|$ stays $\varepsilon$-close to the orbit $P\left(s_{k}\right)$ repeated $k-m$ times (and therefore stays $2 \varepsilon$-close to the orbit $P$ of $f_{r}$ repeated $k-m$ times).

To translate this statement into the language of measures, let us take a continuous function $\varphi:[0,1] \rightarrow \mathbb{R}$. Set

$$
\eta=\sup \{|\varphi(w)-\varphi(v)|:|w-v|<2 \varepsilon\}
$$

and $\ell_{k}=\left|D A^{k} E C\right|=k|A|+|D|+|E|+1$. Then

$$
\ell_{k} \int \varphi d \mu_{Q_{k}}=\sum_{i=0}^{\ell_{k}-1} \varphi\left(f_{s_{k}}(c)\right) \text {. }
$$


This sum can be split into $k-m$ sums, each of them within $|A| \eta$ from $|A| \int \varphi d \mu_{P}$, and a sum of $m|A|+|D|+|E|+1=\ell_{k}-(k-m)|A|$ terms, each of them bounded in absolute value by $\|\varphi\|$ (the $C^{0}$-norm of $\varphi$ ). Therefore

$$
\left|\ell_{k} \int \varphi d \mu_{Q_{k}}-(k-m)\right| A\left|\int \varphi d \mu_{P}\right| \leq(k-m)|A| \eta+\left(\ell_{k}-(k-m)|A|\right)\|\varphi\| .
$$

We divide both sides by $\ell_{k}$ and use notation $\alpha_{k}=(k-m)|A| / \ell_{k}$ :

$$
\left|\int \varphi d \mu_{Q_{k}}-\alpha_{k} \int \varphi d \mu_{P}\right| \leq \alpha_{k} \eta+\left(1-\alpha_{k}\right)\|\varphi\| .
$$

Therefore

$$
\begin{aligned}
\left|\int \varphi d \mu_{Q_{k}}-\int \varphi d \mu_{P}\right| & \leq\left|\int \varphi d \mu_{Q_{k}}-\alpha_{k} \int \varphi d \mu_{P}\right|+\left|\alpha_{k} \int \varphi d \mu_{P}-\int \varphi d \mu_{P}\right| \\
& \leq \alpha_{k} \eta+\left(1-\alpha_{k}\right)\|\varphi\|+\left(1-\alpha_{k}\right)\left|\int \varphi d \mu_{P}\right| \\
& \leq \eta+2\left(1-\alpha_{k}\right)\|\varphi\| .
\end{aligned}
$$

Now we have to define the sequence $\left(r_{n}\right)$. First we take $\varepsilon=1 / n$ and get corresponding $D, E$. Then we take $k$ so large that $\alpha_{k}>1-1 / n$. Note that the numbers $\alpha_{k}$ depend on $D, E$, but not on $\varphi$. Then we set $r_{n}=s_{k}$.

We know already that $\left|r_{n}-r\right|<1 / n$, so $r_{n} \rightarrow r$ as $n \rightarrow \infty$. Moreover, (since $\left.Q_{k}=P_{n}\right)$, we know that

$$
\left|\int \varphi d \mu_{P_{n}}-\int \varphi d \mu_{P}\right| \leq \eta+\frac{2}{n}\|\varphi\| .
$$

The number $\eta$ depends only on $\varepsilon$ (which is $1 / n$ ) and $\varphi$. As $n \rightarrow \infty, \eta$ goes to 0 for a given $\varphi$. Therefore

$$
\lim _{n \rightarrow \infty}\left|\int \varphi d \mu_{P_{n}}-\int \varphi d \mu_{P}\right|=0 .
$$

This holds for all $\varphi$, and therefore the measures $\mu_{P_{n}}$ converge to $\mu_{P}$ in the weak-* topology.

\section{INTEGRALS}

Now we strengthen Theorem 4.4 using known results on the structure of the space of invariant probability measures for logistic maps $f_{r}$. For this we need some notation. We start with measures.

Recall that if $f_{r}$ has an SRB measure (that is, if $r \in \mathcal{S}$ ), we denote this measure by $\sigma_{r}$. If the trajectory of the critical point for $f_{r}$ is a periodic orbit $P$, then we have $\sigma_{r}=\mu_{P}$.

As we mentioned in Section 3, the set of nonwandering points of $f_{r}$ is contained in $M_{r} \cup\{0\}$. Since the only case when 0 can be in the support of $\sigma_{r}$ is $r=4$, and then $0 \in M_{r}$, we restrict our attention to $M_{r}$. Thus, we denote the space of all probability measures invariant for $f_{r}$ with the support contained in $M_{r}$ by $\mathfrak{M}_{r}$. We consider this space with the weak-* topology. We denote by $\mathfrak{P}_{r}$ the subspace of $\mathfrak{M}_{r}$ consisting of 
measures supported on periodic orbits. Thus, if $\mathcal{P}_{r}$ is the set of all periodic orbits of $f_{r}$ contained in $M_{r}$, we have

$$
\mathfrak{P}_{r}=\left\{\mu_{P}: P \in \mathcal{P}_{r}\right\} .
$$

The following theorem is a special case of Theorem 2 from [4] (although perhaps it has been known earlier).

Theorem 5.1 ([4]). If $r \in \mathcal{N}$, then $\mathfrak{P}_{r}$ is dense in $\mathfrak{M}_{r}$.

Now we can generalize Theorem 4.4.

Theorem 5.2. Assume that $r \in \mathcal{N}$ and let $\mu \in \mathfrak{M}_{r}$. Then there is a sequence $\left(r_{n}\right)$ convergent to $r$ such that the $f_{r_{n}}$-orbit $P_{n}$ of $c$ is periodic and the measures $\mu_{P_{n}}$ converge to $\mu$ in the weak-* topology.

Proof. Let $\mathfrak{N}_{r}$ be the set of measures $\nu \in \mathfrak{M}_{r}$ for which there is a sequence $\left(r_{n}\right)$ convergent to $r$ such that the $f_{r_{n}}$-orbit $P_{n}$ of $c$ is periodic and the measures $\mu_{P_{n}}$ converge to $\nu$. By Theorem 4.4, $\mathfrak{P}_{r} \subset \mathfrak{N}_{r}$. Since the space $\mathfrak{M}_{r}$ is metrizable, the set $\mathfrak{N}_{r}$ is closed, so it contains the closure of $\mathfrak{P}_{r}$. By Theorem 5.1, this closure is equal to $\mathfrak{M}_{r}$. Therefore $\mathfrak{N}_{r}=\mathfrak{M}_{r}$.

Now in addition to a dynamical system, we consider an observable. In a general case, if $X$ is a compact metric space and $g: X \rightarrow X$ a continuous map, an observable is a continuous function $\varphi: X \rightarrow \mathbb{R}$. A probability measure $\mu$ on $X$ is invariant if and only if for every observable $\varphi$ we have

$$
\int \varphi \circ g d \mu=\int \varphi d \mu
$$

Lemma 5.3. For $n=1,2, \ldots$, let $g_{n}: X \rightarrow X$ be continuous maps, converging uniformly to $g: X \rightarrow X$. For $n=1,2, \ldots$, let $\mu_{n}$ be a $g_{n}$-invariant probability measure, and assume that $\mu_{n}$ converge to $\mu$ in the weak-* topology. Then $\mu$ is invariant for $g$.

Proof. Let $\varphi: X \rightarrow \mathbb{R}$ be an observable. Then

$$
\int \varphi \circ g_{n} d \mu_{n}=\int \varphi d \mu_{n}
$$

Therefore

$$
\begin{aligned}
\left|\int \varphi \circ g d \mu-\int \varphi d \mu\right| & \leq\left|\int \varphi \circ g d \mu-\int \varphi \circ g_{n} d \mu\right| \\
& +\left|\int \varphi \circ g_{n} d \mu-\int \varphi \circ g_{n} d \mu_{n}\right| \\
& +\left|\int \varphi d \mu_{n}-\int \varphi d \mu\right|
\end{aligned}
$$

Since $\varphi$ is uniformly continuous and $g_{n}$ converge uniformly to $g$, we have

$$
\left|\int \varphi \circ g d \mu-\int \varphi \circ g_{n} d \mu\right| \rightarrow 0
$$


as $n \rightarrow \infty$. Since $\mu_{n} \rightarrow \mu$, we have

$$
\left|\int \varphi \circ g_{n} d \mu-\int \varphi \circ g_{n} d \mu_{n}\right| \rightarrow 0
$$

and

$$
\left|\int \varphi d \mu_{n}-\int \varphi d \mu\right| \rightarrow 0
$$

as $n \rightarrow \infty$. Therefore the right-hand side of (5.1) goes to 0 as $n \rightarrow \infty$, so the left-hand side is equal to 0 . Thus, $\mu$ is invariant for $g$.

Let us add some notation. If $\varphi:[0,1] \rightarrow \mathbb{R}$ is an observable, then we set

$$
\begin{aligned}
S_{r}(\varphi) & =\left\{\lim _{n \rightarrow \infty} \int \varphi d \sigma_{r_{n}}: r_{n} \rightarrow r \text { and }\left(\int \varphi d \sigma_{r_{n}}\right) \text { converges }\right\} \\
T_{r}(\varphi) & =\left\{\int \varphi d \mu: \mu \in \mathfrak{M}_{r}\right\} \\
Q_{r}(\varphi) & =\left\{\int \varphi d \mu: \mu \in \mathfrak{P}_{r}\right\} \\
I_{r}(\varphi) & =\left[\inf Q_{r}(\varphi), \sup Q_{r}(\varphi)\right] .
\end{aligned}
$$

An interesting observation is that the set $T_{r}(\varphi)$ is, according to one of the definitions, the rotation set of the system $\left(M_{r},\left.f_{r}\right|_{M_{r}}, \varphi\right)$.

Theorem 5.4. Assume that $r \in \mathcal{N}$ and let $\varphi:[0,1] \rightarrow \mathbb{R}$ be an observable. Then

$$
S_{r}(\varphi)=T_{r}(\varphi)=\overline{Q_{r}(\varphi)}=I_{r}(\varphi)
$$

Proof. By Theorem 5.2, $T_{r}(\varphi) \subset S_{r}(\varphi)$, and by Lemma 5.3, $S_{r}(\varphi) \subset T_{r}(\varphi)$. Therefore $S_{r}(\varphi)=T_{r}(\varphi)$. By Theorem 5.1, $\overline{Q_{r}(\varphi)}=T_{r}(\varphi)$.

Now, since $\mathfrak{M}_{r}$ is convex and compact, we have $I_{r}(\varphi) \subset T_{r}(\varphi)$. Since $\overline{Q_{r}(\varphi)} \subset I_{r}(\varphi)$, this completes the proof.

\section{Center of mass}

Now we take a concrete simple observable, the identity: $\varphi(x)=x$. Then $\int \varphi d \mu$ can be interpreted as the center of mass of $\mu$. In particular, if $\mu=\mu_{P}$ for a periodic orbit $P$, then

$$
\int \varphi d \mu_{P}=\frac{1}{|P|} \sum_{x \in P} x,
$$

where $|P|$ is the period (cardinality) of $P$. Thus, $\int \varphi d \mu_{P}$ is the center of mass of $P$.

For this observable, we can provide a simple estimate of the interval $I_{r}(\varphi)$.

Lemma 6.1. If $P$ is a periodic orbit of $f_{r}$ then the center of mass of $P$ is smaller than or equal to the largest fixed point of $f_{r}$, which is $(r-1) / r$.

Proof. Denote by $a_{r}$ the largest fixed point of $f_{r}$. Direct computation gives the result $a_{r}=(r-1) / r$. If $a_{r}$ is attracting or neutral, then $f_{r}$ has no periodic orbits other than the fixed points 0 and $a_{r}$, so the claim from the statement of the theorem is true.

Assume now that the fixed point $a_{r}$ is repelling. Then $f_{r}^{\prime}(x)<-1$ for all $x \in$ $\left[a_{r}, f_{r}(c)\right]$. This means that if $x>a_{r}$ then $f_{r}(x)<a_{r}$ and $a_{r}-f_{r}(x)>x-a_{r}$. 
Therefore any periodic orbit $P$ of $f_{r}$ (other than $\left\{a_{r}\right\}$ ) can be divided into blocks of length 2 and 1 with the center of mass of each block smaller than $a_{r}$. Thus, the center of mass of $P$ is smaller than $a_{r}$.

Lemma 6.2. If $f_{r}$ has a periodic orbit $P$ of period 2 , then its center of mass of $P$ is $(r+1) /(2 r)$.

Proof. To find periodic points of period 2, we have to solve the equation

$$
r \cdot r x(1-x) \cdot(1-r x(1-x))-x=0,
$$

that is,

$$
r^{3} x^{4}-2 r^{3} x^{3}+\left(r^{3}+r^{2}\right) x^{2}+\left(1-r^{2}\right) x=0,
$$

and eliminate solutions that correspond to the fixed points. The fixed points are 0 and $(r-1) / r$, so to get an equation for period 2 points we have to divide the left-hand side of the above equation by $x(r x+(1-r))$. We get

$$
r^{2} x^{2}-\left(r^{2}+r\right) x+(r+1)=0 .
$$

If $f_{r}$ has a periodic orbit $P$ of period 2, the points of $P$ are the roots of the above equation, so their sum is $\left(r^{2}+r\right) / r^{2}=(r+1) / r$. Therefore the center of mass of $P$ is $(r+1) /(2 r)$.

From those two lemmas we get the following result.

Proposition 6.3. For the identity observable $\varphi$, if $f_{r}$ has a periodic orbit of period 2 (that is, if $r>3$ ), then the interval $I_{r}(\varphi)$ is nondegenerate. More precisely, its right endpoint is equal to $(r-1) / r$ and its left endpoint is not larger than $(r+1) /(2 r)$.

Proof. The map $f_{r}$ has a periodic orbit of period 2 if and only if the discriminant of the equation (6.1) is positive. A direct computation shows that this is equivalent to $r>3$.

By Lemma 6.1, the right endpoint of $I_{r}(\varphi)$ is $(r-1) / r$. By Lemma 6.2, the left endpoint of $I_{r}(\varphi)$ is not larger than $(r+1) /(2 r)$. If $r>3$ then $(r-1) / r>(r+1) /(2 r)$, so $I_{r}(\varphi)$ is nondegenerate.

From Theorem 3.1 (d) and (e), Theorem 5.4, and Proposition 6.3, we get immediately the main result of the paper.

Theorem 6.4. The family of logistic maps has sensitive dependence on parameters.

\section{Comments}

The idea of this paper is to define sensitive dependence on parameters and provide a natural example of a family with this property. Therefore I did not try to find the largest class of families of interval maps with sensitive dependence on parameters. However, it is clear that any family that shares the properties of the family of logistic maps used in the proof will do. Moreover, we do not need those properties for the "full" interval of parameters, but only locally, for instance in a one-sided neighborhood of the parameter for which the second image of the critical point is the fixed point on the increasing branch (this corresponds to $r=4$ for the logistic maps); to get this is quite easy. 
Moreover, in certain places of the proof I was working with the non-renormalizable maps; generalizations of those results for finitely renormalizable maps without attracting or neutral periodic points is straightforward.

When introducing his notion of chaos, Bob Devaney strengthened the notion of sensitive dependence on initial conditions, by requiring that the set $Y$ from the definition is the whole space. Here I kept the original definition, because requiring a similar thing in the definition of sensitive dependence on parameters (all parameters instead of a set of positive measure) would make the definition useless. Moreover, a set of positive measure is already something "visible" from the "physical" point of view. The probability of a point or parameter being in such a set is positive.

Continuing this idea, one can notice a flaw in Definition 2.3. Namely, for a given parameter $t$ one requires existence of only one parameter $s$ with certain properties, but the measure of the set $\{s\}$ (and thus, the probability that the second parameter will be this $s$ ) is 0 . This means that if we want to get a "visible" sensitive dependence, one should replace $s$ by every parameter from some set of positive measure. This motivates the following definition.

Definition 7.1. The family $\left(X, f_{r}\right)$ has strong sensitive dependence on parameters if there exists an observable $\varphi: X \rightarrow \mathbb{R}$, a set $S \subset T^{\prime}$ of positive measure and $\varepsilon>0$ such that for any $s \in S$ and a neighborhood $U$ of $s$ there is a set $Z \subset U \cap T^{\prime}$ of positive measure such that for every $t \in Z$ we have

$$
\left|\int \varphi d \sigma_{s}-\int \varphi d \sigma_{t}\right|>\varepsilon
$$

Fortunately for us, from Theorem 3.1 (d) and (e), Theorem 5.4, and Proposition 6.3, it follows that the family of logistic maps has strong sensitive dependence on parameters (because small perturbations of the maps for which the critical point is periodic result in maps for which the trajectory of the critical point is attracted to an attracting periodic orbit; the SRB measure changes then continuously).

Note that if our reference measure in the phase space is positive on open nonempty sets (which is normally the case), then a flaw like that does not appear in Definition 2.1. Indeed, if $d\left(f^{n}(x), f^{n}(y)\right)>\varepsilon$, then the same inequality holds if we replace $y$ by another point sufficiently close to $y$.

Another point of view that can be adopted is the "experimental" one. Then "visibility" of some phenomenon means not just occurrence of this phenomenon on a set of positive measure, but on a set of reasonably large measure. This partially saves predictability of long-time averages for the family of logistic maps, because the measure of the set of those $t$ for which (7.1) holds may be very small. In fact, this has been proved by Tsujii [9] for $s=4$. Nevertheless, computer experiments produce pictures in which the center of mass of the SRB measure does not look like a continuous function.

On the other hand, as we mentioned in the introduction, the logistic family with the identity as an observable illustrates another problem with predictions: the change of the long-time averages as the parameter varies is independent of the immediate change. 


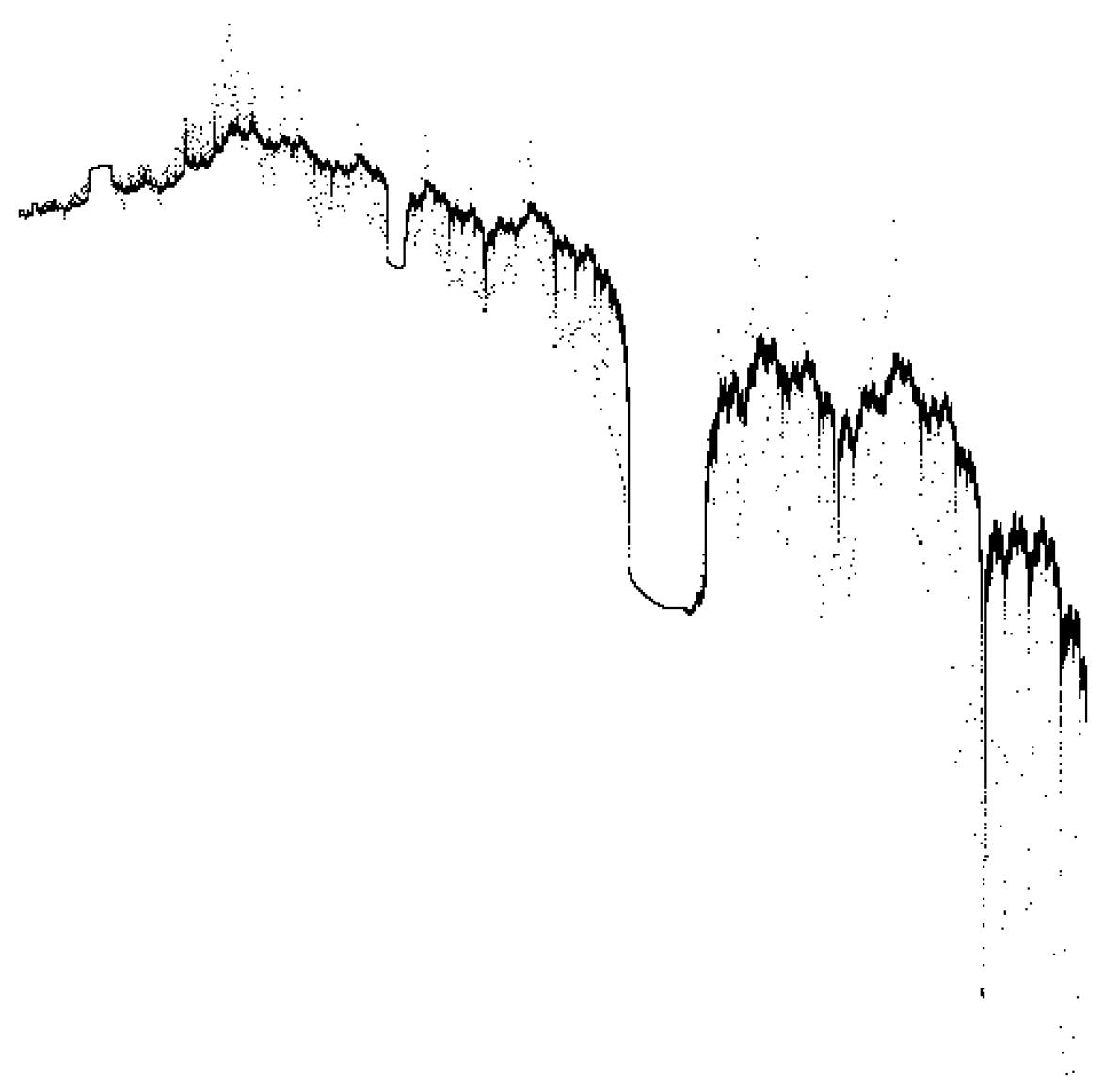

FiguRE 4. The graph of the centers of mass of the SRB measures for the logistic family. One million values of the parameter from 3.6 to 4 were tested.

\section{REFERENCES}

[1] K. Brucks and M. Misiurewicz, The trajectory of the turning point is dense for almost all tent maps, Ergodic Theory Dynam. Systems 16 (1996), 1173-1183.

[2] P. Collet and J.-P. Eckmann, "Iterated maps on the Interval as Dynamical Systems," Birkhäuser, Boston, 1980.

[3] J. Guckenheimer, Sensitive dependence to initial conditions for one dimensional maps, Comm. Math. Phys. 70 (1979), 133-160.

[4] F. Hofbauer and P. Raith, Density of periodic orbit measures for transformations on the interval with two monotonic pieces, Fund. Math. 157 (1998), 221-234.

[5] M. Lyubich, Dynamics of quadratic polynomials III: parapuzzle and SBR measures, Astérisque 261 (2000), 173-200.

[6] W. de Melo and S. van Strien, "One-Dimensional Dynamics," Springer, Berlin, 1993.

[7] D. Ruelle, Dynamical systems with turbulent behavior, in "Mathematical Problems in Theoretical Physics," Springer, Berlin, 1978, pp. 341-360.

[8] M. Rychlik and E. Sorets, Regularity and other properties of absolutely continuous invariant measures for the quadratic family, Comm. Math. Phys. 150 (1992), 217-236.

[9] M. Tsujii, On continuity of Bowen-Ruelle-Sinai measures in families of one dimensional maps, Comm. Math. Phys. 177 (1996), 1-11. 
Department of Mathematical Sciences, iUPui, 402 N. Blackford Street, IndiANAPOLIS, IN 46202-3216, USA

E-mail address: mmisiure@math.iupui.edu 